\title{
Philosophiques
}

\section{Vérité et vérification en logique mathématique et dans les théories physiques}

Yvon Gauthier

Volume 9, numéro 1, avril 1982

URI : https://id.erudit.org/iderudit/203186ar

DOI : https://doi.org/10.7202/203186ar

Aller au sommaire du numéro

Éditeur(s)

Société de philosophie du Québec

ISSN

0316-2923 (imprimé)

1492-1391 (numérique)

Découvrir la revue

\section{Citer cet article}

Gauthier, Y. (1982). Vérité et vérification en logique mathématique et dans les théories physiques. Philosophiques, 9(1), 135-145.

https://doi.org/10.7202/203186ar
Résumé de l'article

Cet article propose une nouvelle approche dans l'analyse et l'interprétation des théories physiques. La théorie des modèles ou sémantique ensembliste est rejetée au profit d'une syntaxe ou théorie des démonstrations qui s'attache d'abord à la structure formelle (logique et mathématique) d'une théorie physique. On donne plusieurs exemples d'une théorie de la preuve (preuve, épreuve, appreuve), exemples qui relèvent surtout de la mécanique quantique et qui vont dans le sens de la thèse principale de l'auteur : la surdétermination de la théorie physique par sa structure mathématique. 


\title{
VÉRITÉ ET VÉRIFICATION EN LOGIQUE MATHÉMATIQUE ET DANS LES THÉORIES PHYSIQUES
}

\author{
par Yvon Gauthier
}

\begin{abstract}
RÉSUMÉ. Cet article propose une nouvelle approche dans l'analyse et l'interprétation des théories physiques. La théorie des modèles ou sémantique ensembliste est rejetée au profit d'une syntaxe ou théorie des démonstrations qui s'attache d'abord à la structure formelle (logique et mathématique) d'une théorie physique. On donne plusieurs exemples d'une théorie de la preuve (preuve, épreuve, appreuve), exemples qui relèvent surtout de la mécanique quantique et qui vont dans le sens de la thèse principale de l'auteur : la surdétermination de la théorie physique par sa structure mathématique.
\end{abstract}

ABSTRACT. This paper deals with a novel approach in the interpretation of physical theories. Model theory or settheoretical semantics is here replaced by a proof-theoretical analysis which is applied to the formal (logical and mathematical) structure of a physical theory. Many examples are given, mainly in Quantum Mechanics, which aim at illustrating the main thesis of the paper: that a physical theory is overdetermined by its mathematical structure and cannot be defined by the class of its empirical structures.

1. La logique classique nous a habitués à la vérité et la notion sémantique de vérité a permis la création d'une théorie des modèles dont on voudrait qu'elle soit l'instrument privilégié de l'interprétation des théories physiques. La sémantique ensembliste de Suppes et Sneed à van Fraassen, par exemple, m'apparaît liée à des «préjugés » réalistes qu'il convient de mettre en doute ou à tout le moins « en suspens». Je propose plutôt une interprétation de la théorie physique en termes de sa structure mathématique, c'est-à-dire syntaxique et non plus en fonction de la classe de ses structures ou sous-structures 
empiriques. Je rappelle quelques notions usuelles : une structure $S$ est un triplet $S=<U_{S}, f_{S}$, pS $>$ où US est l'univers de $S$, fS les fonctions et ps les prédicats de $S$; une sous-structure sera définie par un homomorphisme $h: S \downarrow T$ qui va préserver les fonctions et les prédicats

$$
h\left(f^{S}\left(a_{1}, \ldots, a_{n}\right)\right)=f^{T}\left(h\left(a_{1}\right), \ldots, h\left(a_{n}\right)\right)
$$

et

$$
<a_{1}, \ldots, a_{n}>\in p^{S}, s s i<h\left(a_{1}\right), \ldots, h\left(a_{n}\right)>\in p^{T}
$$

Un isomorphisme est simplement un homomorphisme bijectif. Si l'univers $|T|$ est restreint à l'univers $|S|$, alors $|S|$ et $|T|$ sont isomorphes et par là nous avons une sous-structure. Pour obtenir la notion de structure empirique d'une théorie physique, on n'aura qu'à assouplir un peu cette définition et parler de structures ou modèles partiels (des variations sont possibles ici d'une sémantique ensembliste nä̈ve à la Suppes à une version bourbakiste, mais l'idée est la même). Une sémantique contructiviste est aussi possible, mais au prix de complications que nous n'exposerons pas ici.

La structure syntaxique, l'appareil anallytique comme disait Hilbert, d'une théorie physique est susceptible d'une analyse par des procédures de vérification qui ne garantissent plus la validité d'une théorie (uniquement) par son adéquation empirique, mais aussi par son ancrage mathématique, c'est-àdire sa morphologie et sa syntaxe. Une telle étude n'a rien en commun avec le positivisme logique qui visait " the language of Science ». Par procédures de vérification, j'entends l'examen de la formulation mathématique d'une théorie physique, ses dérivations, sa forme normale, sa normalisation, sa renormalisation, ses réductions, ses extensions. On le voit, il s'agit d'étendre la théorie des démonstrations à l'analyse de la structure mathématique des théories physiques. C'est donc ici le contre-pied de l'approche sémantique et un dépassement de l'approche "linguistique". Vérification veut dire que nous n'avons plus de conditions de vérité, mais des moyens d'évaluer effectivement la validité d'un énoncé ou d'une théorie; ainsi on ne supposera pas que l'on dispose d'un ensemble dénombrable $\boldsymbol{k}_{0}$ de suites dénombrables de nombres 
naturels, mais seulement d'un nombre fini de segments initiaux de telles suites

$$
\varphi(\mathrm{x})<\mathrm{n}, \mathrm{m}, 1 \ldots>[\mathrm{n}, \mathrm{m}, \mathrm{l} \ldots]=1
$$

pour $\mathrm{x}$ un énoncé, $\varphi$ une fonction d'évaluation

$$
\varphi: \text { Form } \rightarrow(0,1)
$$

qui associe des nombres naturels $\mathrm{n}, \mathrm{m}, \mathrm{l}$ aux énoncés ou formules (Form) de la théorie; l'application complémentaire

$$
\xi: \mathrm{N} \rightarrow \mathrm{N}
$$

"identifie" les nombres naturels. On obtient de cette façon une procédure qui permet d'évaluer un énoncé effectivement puisque les $[\mathrm{n}, \mathrm{m}, \mathrm{l}]$ peuvent être considérés comme le nombre d'étapes requis pour vérifier les $\mathrm{n}, \mathrm{m}, \mathrm{l}$. On voit comment une telle démarche s'éloigne de la conception classique de la vérité et de sa logique (classique). Donnons quelques conséquences de ce point de vue.

$1^{\circ} \mathrm{La}$ théorie des démonstrations est essentiellement finitaire.

$2^{\circ}$ L'évaluation des théories physiques et des théories mathématiques est soumise dans les deux cas à des procédures de vérification qui sont analogues et different seulement dans leur généralité.

$3^{\circ}$ La thèse ultime du constructivisme : la physique et les mathématiques ont la même visée, c'est-à-dire la construction du continu.

2. Je propose donc une réorientation de la recherche fondationnelle en physique mathématique qui ne se contente pas d'une sémantique ensembliste élémentaire, mais qui exploite toutes les ressources d'une logique mathématique (théorie des démonstrations ici) capable de s'adapter à son objet et en même temps susceptible de dépasser les exemples simplistes de l'analyse traditionnelle (e.g. axiomatisation de la mécanique classique "ponctuelle» chez Sneed). Prenons les actuelles théories de jauge, celle de Weinberg-Salam, par exemple; les problèmes de renormalisation qu'on a réussi à résoudre et ceux qui ne sont pas encore résolus pour des théories plus larges (e.g. la supergravitation), les sous-systèmes de variétés différentiables pour les champs de Yang-Mills et les 
équations du champ d'Einstein ou encore l'extension de la géométrie complexe (algébrique) dans le programme de Penrose. Bien entendu, cette étude devrait mettre en œuvre des moyens énormes qui transcendent les outils ordinaires du philosophe des sciences qui n'a souvent qu'une connaissance rudimentaire de la physique et des mathématiques, mais c'est le prix qu'il faut payer pour ne pas être confiné dans des analyses linguistiques triviales et qui intéressent peu le physicien et le mathématicien. L'épistémologicien dans sa tâche d'analyse et de critique fondationnelles ne se substitue pas à ces derniers, il les suppose et les supporte, peut-on dire. C'est que son travail est postérieur à celui du scientifique et s'il arrive qu'il le devance, c'est parce qu'il aura trouvé une voie heuristique que son recul par rapport à la pratique d'une science lui aura permis de repérer. Quoi qu'il en soit, il doit adopter le langage du scientifique, au moins dans une première étape, s'il veut se faire entendre, puisque c'est la communauté scientifique at large qui est la cour de dernière instance en ces matières, à moins que l'on veuille faire de la philosophie pour les philosophes, ce qui est une autre affaire.

J'en viens maintenant aux conséquences que j'ai indiquées plus haut. C'est Hilbert, lui qui a créé la théorie des démonstrations, Beweistheorie qu'il appelait aussi Metamathematik, qui avait raison lorsqu'il insistait sur le caractère fini (finit) des démonstrations et sur la sûreté (Sicherheit) qu'elles devaient permettre. On sait quelles modifications le programme hilbertien de la métamathématique comme combinatoire finie a subies avec les résultats de Gödel; mais on ne peut pas supposer avec Gödel, après 1931, que seules les méthodes infinitaires peuvent résoudre le problème de la noncontradiction de l'analyse classique (y compris la théorie des ensembles). Parce que les concepts sémantiques (c'est-à-dire de la théorie des modèles) sont souvent indéterminés - ce que Skolem avait bien vu - on ne peut espérer aller beaucoup plus loin dans ce sens. Les piétinements de la théorie des modèles (et de la sémantique ensembliste) depuis vingt ans malgré les efforts d'une armée de chercheurs à la poursuite de cardinaux « évasifs » en témoignent abondamment. D'autre part, la théorie des démonstrations a fait des emprunts avec l'induction 
transfinie à l'arithmétique cantorienne mais sans emporter l'adhésion. On peut mettre d'autres moyens en œuvre, que j'appelle "effinitaires", mais ce n'est pas le moment de les exposer ici. Je voudrais plutôt insister, avant de passer à mon second point, sur les trois composantes d'une démonstration : ce sont, pour moi, la preuve, l'épreuve et l'appreuve ou l'approbation. On peut avoir une preuve - qui doit être un "texte " même si c'est un objet mental comme le veut l'intuitionnisme - qui soit imparfaite ou incomplète; l'épreuve pourra la compléter, la parfaire, la simplifier, l'abréger souvent ; enfin, l'appreuve signifie l'acceptation des moyens de preuve par la communauté des chercheurs. Un bon exemple est la conjecture des quatre couleurs démontrée récemment par Appel et Haken; certains mathématiciens voudraient avoir une preuve "faite à la main " - c'est l'épreuve - parce qu'ils ne se fient pas totalement à l'ordinateur et à la longueur de ses preuves - c'est l'appreuve. Je donne cet exemple parce qu'il a entraîné chez quelques-uns des spéculations sur le caractère empirique de la preuve. Appel, par exemple, voudrait qu'on accepte la vérité du dernier théorème de Fermat puisque la probabilité qu'il soit vrai est très grande, si on se fonde sur les calculs déjà faits. C'est là un point de vue indéfendable; la vérité probable ne constitue en fait qu'un « contre-contre-exemple » et sa force démonstrative se limite à un cas singulier

$$
\text { In } \forall x \forall y \quad \forall z\left(x^{n}+y^{n} \neq z^{n}\right) \text { pour } n>2 .
$$

La notion de probabilité, en dehors de la théorie mathématique des probabilités, a un sens subjectif ou bayésien que je ne veux pas discuter ici mais qui lui interdit de jouer un rôle dans la logique de la démonstration si ce n'est dans la logique de l'induction.

3. Une théorie physique n'est pas vraie au même titre qu'une théorie mathématique et devrait donc être sujette à des procédures de vérification différentes, sinon divergentes. Je suppose cependant que les trois moments que j'ai décrits plus haut, preuve, épreuve, appreuve s'appliquent aussi bien en physique qu'en mathématiques. Évidemment, la preuve n'a plus le sens en physique d'une démontration à l'aide de règles d'inférence ou de déduction, mais la corroboration qui n'est 
pas concluante doit comporter un nombre fini d'instances, l'épreuve est l'ensemble des "tests" que doit passer la théorie et l'appreuve est l'approbation des moyens techniques et de la valeur scientifique de la théorie. Prenons un exemple suffisamment récent : l'électrodynamique quantique de Schwinger et Feynman. C'est une très bonne théorie dans le sens que son adéquation aux expériences est très grande, qu'elle obtient un haut degré d'approximation. Dans ce cas, la preuve comporterait la vérification de la consistance et de la complétude théorique de la théorie, l'épreuve la confrontation avec les résultats expérimentaux et l'appreuve viendrait avec la procédure de renormalisation (à l'aide des diagrammes de Feynman ici) qui justifie les deux premières étapes. Cette justification ou certification (Sicherung) n'a pas un caractère subjectif, elle repose sur des moyens de preuve qui peuvent être essentiellement différents, e.g. méthodes analytiques ou transcendantales et méthodes arithmétiques ou constructives (ou encore élémentaires) en théorie des nombres. L'épreuve n'étant jamais exhaustive puisqu'il n'existe pas de règle de fermeture catégorique ou canonique pour une théorie physique, la théorie physique ne sera jamais vérifiée complètement au sens d'un théorème mathématique dont la démonstration par cas (induction mathématique) doit être complète, mais sera toujours affectée d'un coefficient d'indétermination, c'est-à-dire d'un degré de probabilité (e.g., la relativité restreinte a un degré de probabilité de .9 si on veut).

4. Ce que je viens de dire ne signifie pas que physique et mathématiques sont irrémédiablement séparées, mais seulement que les constructions mathématiques sont plus générales ; elles dessinent les formes du possible qui ne sont pas isomorphes a priori aux formes du réel, les deux ordres de construction du monde, mathématique et physique, convergent cependant dans une unification jamais achevée. Mon troisième thème aborde ce rapport et le problème, qui est passé autrefois pour un mystère, de l'applicabilité des mathématiques au monde physique.

Je veux prendre l'exemple de la mécanique quantique pour étayer mon point de vue. Prenons les axiomes «physiques» de la $\mathrm{MQ}$ : 
$1^{\circ}$ Les états du système physique sont représentés par des vecteurs d'état (dans un espace de Hilbert) ;

$2^{\circ}$ Il y a bijection entre les observables et les opérateurs hermitiques ("dogme " de von Neumann);

$3^{\circ}$ L'évolution d'un système physique est décrite par l'équation temporelle de Schrödinger ;

$4^{\circ} \mathrm{La}$ probabilité de trouver une (ou des) particule(s) dans une position particulière est donnée par $\operatorname{prob}(\mathrm{r}, \mathrm{t})=\psi^{*}(\mathrm{r}, \mathrm{t}) \psi(\mathrm{r}, \mathrm{t})=|\psi(\mathrm{r}, \mathrm{t})|^{2}$ où $r$ est le vecteur de position, $\psi^{*}$ le conjugué complexe de $\psi\left(\psi^{*} \psi=|\psi|^{2}\right)$.

On pourrait ajouter ici un cinquième postulat (dû à von Neumann), soit le postulat de projection qui stipule qu'immédiatement après une mesure (c'est-à-dire une interaction), la superposition $\Sigma c_{j} \sigma_{j} \alpha_{j}$ se transforme ou est réduite en $\sigma_{n} \alpha_{n}$ (réduction du paquet d'ondes).

Il serait erroné de voir dans ces axiomes un contenu physique "pur". On sait en effet qu'ils "découlent " des axiomes mathématiques de l'espace de Hilbert dans la formulation de von Neumann. Un espace de Hilbert $\Psi$ est un espace vectoriel linéaire à coefficients complexes qui possède un produit intérieur strictement positif, est séparable et complet ; un espace de Hilbert est un espace métrique et par là un espace topologique. L'intérêt "physique " de l'espace de Hilbert, c'est qu'on peut y définir une notion d'orthogonalité

$$
(\mathrm{f}, \mathrm{g})=0
$$

qu'on écrit $\mathrm{f}^{\mathrm{L}} \mathrm{g}$; le complément orthogonal de $\mathrm{f}$, $\mathrm{f}^{\mathrm{L}}$ obéit à la loi booléenne $f^{\mathrm{L} L \mathrm{~L}}=\mathrm{f}$ et $\mathrm{f}^{\mathrm{I}}$ constitue un sous-espace, c'est-à-dire une variété linéaire fermée de $\Psi$; il est important de noter que pour la physique quantique les sous-espaces sont en bijection avec les projections, c'est-à-dire les opérateurs linéaires $\mathrm{E}$ tels que $\mathrm{EE}^{*}=\mathrm{E}$ pour $\mathrm{E}^{*}$ l'adjoint de $\mathrm{E}$ pour lequel $\left(\mathrm{E}^{*}\right)^{*}=\mathrm{E}-$ si $\mathrm{E}^{*}=\mathrm{E}$, alors $\mathrm{E}$ est auto-adjoint ou hermitique. Le théorème spectral énonce qu'il y a une bijection entre les opérateurs auto-adjoints et les mesures spectrales sur la droite réelle et ce qu'on peut appeler le dogme de von Neumann affirme qu'il y a une bijection entre opérateurs auto-adjoints et observables ou grandeurs physiques (notre 
deuxième axiome). On voit aisément que ce deuxième postulat de la MQ est issu en droite ligne du théorème spectral qui renvoie lui-même au théorème de Riesz-Fischer en analyse fonctionnelle et qui a été inspiré par les travaux de Hilbert sur les équations intégrales. Certains, comme Jauch, vont même jusqu'à dire, par abus de langage, que les observables sont les opérateurs linéaires auto-adjoints. ${ }^{1}$ Mais en 1952, Wick, Wightman et Wigner ont introduit des règles du supersélection montrant qu'il existe des opérateurs hermitiques qui ne correspondent pas à des observables, pratiquant ainsi la première brèche dans le dogme de von Neumann ; par ailleurs, Park et Margenau, entre autres, ont soutenu qu'il existe des observables, par exemple, les composantes non commutatives $\mathrm{x}$ et $\mathrm{z}$ du spin (d'un électron) qui n'ont pas d'opérateurs hermitiques pour les représenter. La bijection coule donc par les deux bouts. . . ${ }^{2} \mathrm{Ce}$ que cet exemple montre, c'est que l'appareil analytique de la théorie physique, son armature mathématique, surdétermine ses modèles et que souvent, et de plus en plus en physique contemporaine, la structure mathématique construit (homomorphiquement) des modèles que la recherche empirique ne saurait remplir. La syntaxe mathématique (et logique) d'une théorie physique rend possible son adéquation empirique, ce n'est pas la classe des modèles empiriques d'une théorie physique qui rend compte de sa structure mathématique.

Revenons à l'orthogonalité dans un espace de Hilbert ; le complément orthogonal $\mathrm{f}^{\mathrm{L}}$ correspond à l'orthocomplément $\mathrm{a}^{-}$ d'un treillis booléen défini par

$$
\left(a^{-}\right)^{-}=a, a^{-} \wedge a=\phi \text { et } a \leqslant b\left\langle b^{-} \leqslant a^{-}\right. \text {. }
$$

L'orthocomplémentation induit un antiautomorphisme involutif $\left(\mathrm{a}^{*}\right)^{*}$ sur le champ de vecteurs d'un espace vectoriel. C'est un tel antiautomorphisme qui sous-tend l'important théorème de Gleason (1957) qui démontre que toute mesure de probabilité $\mu(A)$ sur les sous-espaces de $\Psi$ a la forme

$$
\mu(A)=\operatorname{Tr}\left(W P_{A}\right)
$$

1. Cf. J.M. Jauch Foundations of Quantum Mechanics, Addison-Wesley. Reading, Mass., 1968 , p. 132.

2. Je renvoie pour cette question à mon article "The Use of the Axiomatic Method in Quantum Physics», Pbilosopby of Science, vol. 38, no. 3, (1971), pp. 429-437. 
où $\operatorname{Tr}$ signifie $\operatorname{Tr} X=\Sigma_{r}\left(\varphi_{r}, X_{\varphi_{r}}\right)$ pour tout système complet de vecteurs orthogonaux normalisés $\varphi_{r} ; P_{A}$ dénote la projection orthogonale de $\mathrm{A}$ et $\mathrm{W}$ est un opérateur hermitique qui vérifie

$$
\mathrm{W}>0, \operatorname{Tr} \mathrm{W}=1 \text { et } \mathrm{W}^{2} \leq \mathrm{W} .
$$

Kochen et Specker ont obtenu en 1967 un résultat intimement lié au théorème de Gleason et qui montre que dans un espace de plus de deux dimensions il ne peut y avoir d'homomorphisme bi-valué $h: A \longrightarrow A^{\prime}$ de l'algèbre $A$ des opérations partielles sur les observables compatibles d'une algèbre booléenne commutative $A^{\prime}-A$ n'est donc pas plongeable dans une algèbre booléenne.

De tels résultats ont donné un nouvel essor à la logique quantique chez des Finkelstein, Putnam, Bub, Demopoulos, etc. J'ai proposé récemment de substituer à l'algèbre booléenne partielle une algèbre de Heyting ou pseudo-booléenne partielle dans laquelle le complément relatif ou local remplace le complément booléen; pour un treillis $\mathrm{B}$, un élément $\mathrm{c}$ de $\mathrm{B}$ est le pseudo-complément de a relatif à $b$, si c'est le plus grand élément tel que

$$
a<c \leqslant b
$$

c'est donc le sous-ensemble le plus grand qui differe de a et nous avons

$$
a \leqslant 77 a .
$$

Une telle «localisation» du complément permet de montrer comment l'ensemble de tous les sous-ensembles d'un espace de Hilbert n'est pas orthocomplémentable (même dans le cas d'un espace de dimension finie) et comment on pourrait faire une «place » mathématique à l'observateur dans la MQ. ${ }^{3}$

5. Je pourrais donner d'autres exemples pour illustrer mon point de vue (e.g. la théorie chronogéométrique de I.E. Segal $)^{4}$, mais ils déborderaient le cadre de cet exposé. Je

3. Voir là-dessus mon arricle "Quantum Mechanics and the Local Observer " à paraître dans The International Journal of Theoretical Pbysics.

4. Cf. I.E. Segal Mathematical Cosmology and Extragalactic Astronomy. Academic Press, New York, 1976. 
termine donc avec quelques remarques sur le problème de la mesure dans la $M Q$, problème qui est encore très actuel, surtout en Europe, si l'on songe à l'intérêt provoqué par l'inégalité de Bell. Ce nouveau paradoxe est parent du célèbre paradoxe d'Einstein-Podolski-Rosen. Sans aller jusqu'à invoquer l'intégrité, «wholeness », du monde quantique comme le font certains, on peut remarquer qu'ici encore le paradoxe ne sera pas résolu simplement en abandonnant l'un des principes de la MQ, le principe de localité par exemple. Je pense qu'il faut ici aussi s'interroger sur la structure logico-mathématique de la théorie physique et proposer peut-être (à la suite de Bohr) que la mesure ou l'observateur modifie la situation phénoménale originelle de telle sorte que les mesures orthogonales de probabilité (ou les mesures sur les sous-espaces orthogonaux) ne sont pas conservées, puisque l'effet de la mesure (ou de l'observateur local) est d' "ouvrir" l'espace de Hilbert des observables, c'est-à-dire d'en faire un espace qui n'est pas orthocomplémentable. Quoi qu'il en soit, ce n'est pas en ressuscitant l'interprétation des "multivers" de Everett, que certains invoquent sans se rendre compte de son inconsistance, qu'on pourra faire avancer la question; dans la formulation d'Everett, la fonction d'onde $\psi$ doit prendre toutes ses valeurs dans la ramification complète de l'équation d'onde universelle, ce qui signifie qu'elle doit prendre $2 \aleph_{0}$ (cardinalité de $\mathrm{R}$ ou $\mathrm{C}$ ) valeurs, mais il n'y a certainement pas plus de $\boldsymbol{\kappa}_{\mathbf{0}}$ mesures ou résultats de mesure possibles : il n'y a pas de bijection entre $\boldsymbol{\kappa}_{0}$ et $2 \boldsymbol{\aleph}_{0}$ et si l'on tient compte de la thèse d'Everett sur l'isomorphisme entre le formalisme et l'interprétation de la $M Q$, cela suffit à réfuter l'interprétation des univers multiples. ${ }^{5}$

Il faut donc plus de souplesse et plus de rigueur formelle (et informelle) à la fois, pour évaluer critiquement les théories physiques. Je ne pense pas avoir démontré ma thèse, à savoir que les mathématiques et la physique visent ultimement toutes deux la construction du continu, mais j'en ai donné quelques jalons, surtout ce que j'ai appelé la surdétermination du physique par le mathématique. Les exemples que j'ai donnés

5. Pour ces questions, voir mon ouvrage Théorétiques. Pour une philosophie constructiviste des sciences, à paraître. 
relèvent tous de la mathématique du continu (la physique du discontinu, malgré les apparences, ne peut être traitée que par une mathématique «continuiste »). C'est l'analyse logicomathématique de l'appareil analytique des théories physiques qui devra permettre de démonter les mécanismes de l'application des structures mathématiques au monde physique beaucoup plus que les thèses philosophiques générales des scientifiques et des philosophes sur la construction mathématique du réel physique.

Département de philosophie Université de Montréal 